\title{
Fetal supraventricular tachycardia at 12 weeks of gestation: diagnosis and follow up. A case report
}

\author{
Nathalie Jeanne Bravo-Valenzuela ${ }^{1}$, Alberto Borges Peixoto ${ }^{1,2,3}$, Gabriele Tonni ${ }^{4}$, \\ Edward Araujo Júnior ${ }^{1}$
}

${ }^{1}$ Department of Obstetrics, Paulista School of Medicine-Federal University of São Paulo (EPM-UNIFESP), São Paulo, Brazil, ${ }^{2}$ Department of Obstetrics and Gynecology, University of Uberaba (UNIUBE), Uberaba-MG, Brazil, ${ }^{3}$ Department of Obstetrics and Gynecology, Federal University of Triângulo Mineiro (UFTM), Uberaba-MG, Brazil, ${ }^{4}$ Prenatal Diagnostic Service, Department of Obstetrics and Gynecology, AUSL Reggio Emilia, Italy.

\begin{abstract}
This report describes a case of fetal supraventricular tachycardia (SVT) diagnosed at 12 weeks of gestation in a pregnant woman with diabetes mellitus. Transplacental digoxin therapy administered orally to the mother was unsuccessful. Subsequently, sotalol was added to digoxin to achieve fetal heart rate (HR) control and the conversion to sinus rhythm was achieved. The fetal HR remained stable until term, and a healthy male baby was born. The newborn electrocardiogram showed sinus rhythm with normal PR and QTc intervals. When the newborn was stable, he was discharged with propanolol. Sustained SVT is extremely rare during the first trimester. The goal of treatment in utero is the conversion to sinus rhythm or reduction of the ventricular rate to tolerable levels, preventing or even reversing fetal hydrops.
\end{abstract}

Keywords: prenatal diagnosis; arrhythmias; ultrasound imaging; echocardiography

\section{Introduction}

In general, fetal arrhythmias are detected during the second or third trimester routine obstetric ultrasound and occur in up to $2 \%$ of all pregnancies [1]. Most fetal arrhythmias are benign and transient; however, some of them, such as sustained tachycardia (up to 200 beats per minute $[\mathrm{bpm}]$ ), may result in low cardiac output, fetal hydrops and fetal demise. Supraventricular tachycardia (SVT) is the most common type of tachycardia and a frequent cause of non-immune hydrops [2].

Cardiac ultrasound/echocardiography can be used to assess the fetal cardiac rhythm, enabling the differential diagnosis of the majority of tachyarrhythmias. The ven-

Received 18.04.2018 Accepted 21.06.2018

Med Ultrason

2019, Vol. 21, No 1, 93-95

Corresponding author: Prof. Edward Araujo Júnior, $\mathrm{PhD}$

Rua Belchior de Azevedo, 156 apto. 111

Torre Vitoria

São Paulo-SP, Brazil, CEP 05089-030

Phone/Fax: +55-11-37965944

E-mail: araujojred@terra.com.br triculoatrial (VA) and atrioventricular (AV) time intervals should be measured by M-mode and Doppler ultrasound [3]. The AV and VA intervals are mechanical analogs of the PR and RP electric intervals of the ECG. The short VA type of SVT is more common than long VA sinus tachycardia (ST), which is more difficult to diagnose and treat in utero. Ectopic atrial tachycardia and permanent junctional reciprocating tachycardia are long VA types of SVT. Cardiac ultrasound provides a way to assess the hemodynamic consequences of the tachyarrhythmias, aiding the detailed analysis of the fetal cardiac anatomy. Ebstein's anomaly, cardiac tumors, and myocarditis should be excluded.

The authors describe a rare case of SVT, with no hydrops, diagnosed at 12 weeks of gestation. Despite the earlier presentation and the long VA interval, the combination therapy with digoxin and sotalol was successful in treating the SVT.

\section{Case report}

A 39-year-old woman, gravida 5 para 4, was referred for fetal echocardiography because of fetal SVT 


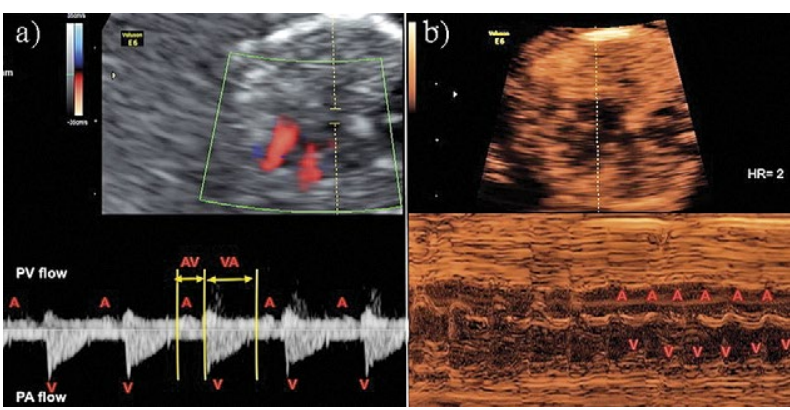

Fig 1. a) Fetal echocardiogram performed at 12 weeks of gestation showing SVT. The simultaneous pulmonary vein and pulmonary artery pulse wave Doppler tracings demonstrated tachycardia with 1:1 AV conduction and long VA interval time. Measurements of the AV and VA intervals are illustrated by vertical bars and arrows; b) Dual or anatomic M-mode ultrasound showing SVT. Mmode guided by real time 2D-imaging was aligned to obtain the atrial and ventricular wall motions simultaneously. Note the 1:1 AV relationship with fast HR. SVT: supraventricular tachycardia; A: atrial contraction; V: ventricular contraction; HR: heart rate; VA: ventricular-atrial interval time; AV: atrioventricular interval time; PV: pulmonary vein flow; PA: pulmonary artery flow

$(>200 \mathrm{bpm})$ noted in a routine obstetric ultrasound performed at 11 weeks of gestation. There was no history of infectious diseases, thyrotoxicosis, or consumption of any drugs. The patient was diabetic and insulin dependent.

The transvaginal fetal echocardiogram confirmed the tachycardia with fetal heart rate (HR) ranging from 215 to $239 \mathrm{bpm}$ with 1:1 AV relationship and long VA interval $(\mathrm{VA} / \mathrm{AV}$ ratio $=3.0$; VA interval time $=223 \mathrm{msec})($ fig 1$)$. The fetal echocardiogram showed normal cardiac anatomy and no signs of hydrops. -

Maternal electrocardiogram (ECG) and renal function results were normal and oral digoxin was started at $2.0 \mathrm{mg} /$ day, reduced every $24 \mathrm{hrs}$ to $1.5 \mathrm{mg}$ and $1.0 \mathrm{mg} /$ day. Because of the persistence of fetal SVT, sotalol $(320 \mathrm{mg} /$ day) was added to digoxin ( $0.25 \mathrm{mg}$ twice daily). Sotalol was suspended due to the prolongation of the maternal QT interval and the patient was discharged with digoxin. Subsequently, as the fetus maintained the SVT and maternal ECG QT interval was normalized, sotalol was once again added ( $240 \mathrm{mg} /$ day) to digoxin. The conversion to sinus rhythm was achieved by weeks 20 onwards and the fetal HR remained stable until 39 weeks of gestation. The male baby was born healthy, weighing $3675 \mathrm{~g}$, with Apgar scores of 9 and 10 at 1 and $5 \mathrm{~min}$, respectively. The neonatal electrocardiogram and echocardiogram were normal and he was discharged with propranolol therapy regimen.

\section{Discussions}

Tachyarrhythmias are divided into three categories: ST, SVT (atrial flutter, atrial ectopic tachycardia, and conduction type tachycardias), and ventricular tachycardia (VT). SVT is the most common type of tachycardia with VT as the rarest type [1,4].

In general, arrhythmias are detected from the second trimester of pregnancy onwards being more frequently after 25 weeks of gestation [5]. Rarely, SVT has early onset and has been diagnosed as early as 13 weeks of gestation [6].

Although fetal magnetocardiography is an effective and promising non-invasive technique to assess fetal cardiac electrical impulses, cardiac Doppler ultrasound is currently the most commonly used tool in a clinical setting [7]. The assessment of the relationships between atrial and ventricular contractions is crucial for an accurate diagnosis of fetal arrhythmias by using M-mode and Doppler ultrasound (fig 2). The M-mode technique can be used to obtain better quality recordings as early as the first trimester, especially in cases of unfavorable fetal position or increased body mass index in the mother.

In ST, atrial and ventricular rates are similar (1:1 AV relationship), and the HR is more variable and lower (at or below $200 \mathrm{bpm}$ ) than in SVT. Re-entrant SVT is the most common type of SVT, where the HR usually ranges between 220 and $300 \mathrm{bpm}$, with 1:1 AV and short A-V time interval. The anatomical substrate of re-entrant SVT is the presence of an accessory electrical conduction pathway. As the retrograde conduction is fast, the atrium is excited shortly after the ventricle, which generates a short VA time interval on Doppler or M-mode ultrasound $(\mathrm{VA}<\mathrm{AV})$ with a fast HR. SVT with longer VA interval (VA $>$ AV) includes atrial ectopic tachycardia and persistent junctional reciprocating tachycardia (PJRT) [8]. PJRT is a rare type of re-entrant SVT in which the retrograde pathway has a slow conduction leading to a characteristic long AV interval with 1:1 AV. In atrial ectopic tachycardia, the mechanism is related to an atrial automatic focus with 1:1 AV relationship and variable HR (ranging from 160 to $250 \mathrm{bpm}$ ). Because of the longer AV interval, PJRT or atrial tachycardia are the possible types of SVT considered in this case report.

Another type of SVT is junctional tachycardia (JET) which is extremely rare in utero,. It is caused by an automatic ectopic focus in the $\mathrm{AV}$ junction in which the $\mathrm{A}$ wave is superimposed on the $\mathrm{V}$ wave. Fetal JET and AF have been reported to be related to Anti-Ro (SSA) and La (SSB) isoimmunization. AF is a type of SVT caused by an intra-atrial re-entry circuit with atrial rate higher than ventricular rate (atrial rate ranging from 350 to $500 \mathrm{bpm}$ ), usually with a $2: 1$ ratio. It is observed only in the third trimester of pregnancy $[4,5]$.

For treatment in utero, antiarrhythmic medications are recommended for all sustained SVT, VT, intermittent SVT with hydrops, and VT with HR $>200 \mathrm{bpm}$. The only 


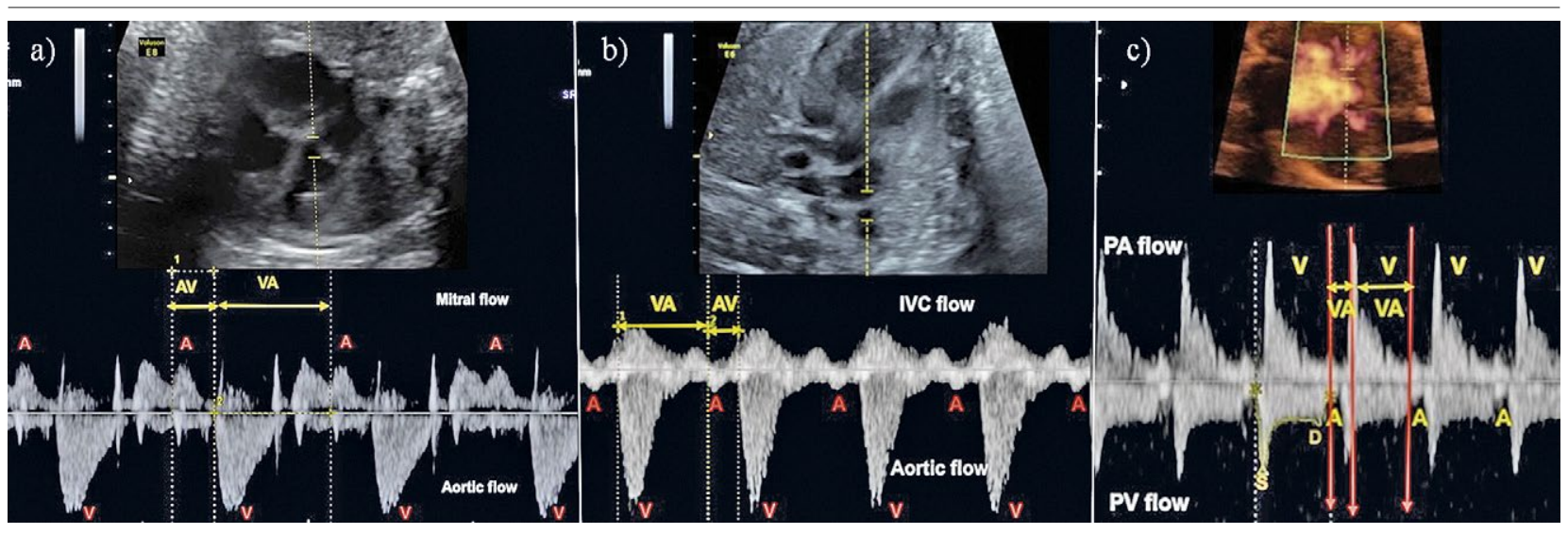

Fig 2. Simultaneous recordings of atrial and ventricular flow waves by Doppler ultrasound/echocardiography showing how to measure the AV and VA intervals by (A) inflow and outflow ventricular waves (four-chamber view); (B) superior vena cava and aortic Doppler flow; (C) pulmonary vein and pulmonary artery Doppler waves (four-chamber view). M: mitral Doppler wave (left ventricular inflow); Ao: aortic Doppler wave (left ventricular outflow); SVC: superior vena cava flow; PV: pulmonary vein flow wave; PA: pulmonary artery flow wave; A: atrial contraction; V: ventricular contraction; VA: ventriculo-atrial interval time; AV: atrioventricular interval time.

exception is for near term fetuses with SVT/VT. Antiarrhythmic medications are used transplacentally in the pregnant woman (given orally or intravenously) or in the fetus. Currently, digoxin is still the first-line therapy in many centers to treat fetuses with fetal SVT mainly in short AV SVT. In other centers, flecainide or sotalol have been used as first-line therapy [5].

A recent systematic review and meta-analysis demonstrated that flecainide is more effective than digoxin in treating SVT with fewer adverse effects [9]. In refractory SVT, amiodarone has been used as second- or third-line therapy alone or in combination with digoxin. In the case presented, the initial therapy was digoxin because flecainide was unavailable in our country. Subsequently, as amiodarone is contraindicated during the first trimester because of the side effect of fetal/neonatal hypothyroidism, a combination therapy with sotalol and digoxin was used to treat SVT in the reported case, with a successful outcome. Interestingly, postnatal recurrence of SVT during the first year of life has been described (10-20\% of SVT) [10]. Therefore, the drug regimen was continued as a prophylactic to prevent recurrence of SVT in this case.

In summary, sustained SVT is extremely rare during the first trimester of pregnancy. The goal of treatment in utero is the conversion to sinus rhythm or reduction of the ventricular rate to tolerable levels thereby preventing or even reversing fetal hydrops.

\section{References}

1. Hornberger LK, Sahn DJ. Rhythm abnormalities of the fetus. Heart 2007;93:1294-1300.
2. Gest AL, Bair DK, Vander Straten MC. Thoracic duct lymph flow in fetal sheep with increased venous pressure from electrically induced tachycardia. Biol Neonate 1993;64:325-330.

3. Jaeggi E, Fouron JC, Fournier A, van Doesburg N, Drblik SP, Proulx F. Ventriculo-atrial time interval measured on $\mathrm{M}$ mode echocardiography: a determining element in diagnosis, treatment, and prognosis of fetal supraventricular tachycardia. Heart 1998;79:582-587.

4. Krapp M, Kohl T, Simpson JM, Sharland GK, Katalinic A, Gembruch U. Review of diagnosis, treatment, and outcome of fetal atrial flutter compared with supraventricular tachycardia. Heart 2003;89:913-917.

5. Donofrio MT, Moon-Grady AJ, Hornberger LK, et al. Diagnosis and treatment of fetal cardiac disease: a scientific statement from the American Heart Association. Circulation 2014;129:2183-2242.

6. Porat S, Anteby EY, Hamani Y, Yagel S. Fetal supraventricular tachycardia diagnosed and treated at 13 weeks of gestation: a case report. Ultrasound Obstet Gynecol 2003;21:302-305.

7. Batie M, Bitant S, Strasburger JF, Shah V, Alem O, Wakai RT. Detection of Fetal Arrhythmia Using Optically-Pumped Magnetometers. JACC Clin Electrophysiol 2018;4:284287.

8. Fouron JC. Fetal arrhythmias: the Saint-Justine hospital experience. Prenat Diagn 2004;24:1068-1080.

9. Alsaied T, Baskar S, Fares M, et al. First Line Antiarrhythmic Transplacental Treatment for Fetal Tachyarrhythmia: A Systematic Review and Meta Analysis. J Am Heart Assoc 2017;6:e007164.

10. Hansmann M, Gembruch U, Bald R, Manz M, Redel DA. Fetal tachyarrhythmias: transplacental and direct treatment of the fetus - a report of 60 cases. Ultrasound Obstet Gynecol 1991;1:162-168. 